\title{
A Low Cost Design of the Rural Intelligent Meter Reading System
}

\author{
Huishan Yu, Chuankao Yao \\ School of physical sciences and information engineering, Liao Cheng University, Liao Cheng, Shan Dong Province, China \\ yuhuishan@1cu.edu.cn, yck871@126.com
}

\begin{abstract}
Analysis of the current use of rural electricity meters, and according to the situation of economic development in rural areas of China, to the greatest extent to reduce costs as the goal, intelligent remote meter reading system is presented which is applicable to China's rural areas. The system is composed of electric energy meter, collector, concentrator, the remote control center. Between collectors and concentrators use low voltage power line carrier communication, concentrator through the GSM network and remote control centre for internet. The collector and concentrator use AT89C52 as control chip, which reduces the system cost.

Index Terms - Intelligent meter reading system, Low-voltage power line carrier, GSM, AT89C52
\end{abstract}

\section{Introduction}

At present, each home has an electric meters in the rural areas of China, using the traditional hand-written manual mode is not only time-consuming, laborious, but also may cause phenomena of drain copy, misuse copy, copy estimate [1]. The smart metering system not only overcome these shortcomings, but also can monitor the energy use for each user, understand the situation, such as load power supply of the network, as well as power line network failure in 24 hours. Therefore, how build a suitable smart meter reading system with minimum cost in rural areas is an urgent problem. This paper presents a lowcost smart meter reading system suitable for rural areas, but many users still use old-fashioned mechanical meters. It was costly if replaced all the mechanical meters with carrier or electronic meters. Therefore, it is need to install groove coupler in the old-fashioned mechanical to acquire pulse signal for smart metering.

\section{The Overall System Design}

The meter reading system is consist of the meter, the collector (collecting and storing electricity of multiple meters, as well as receiving control command from concentrator to upload the collected data), concentrator (receiving control command from the control center and uploading the data of electricity to the remote control center) and remote control Center (responsible for the entire power management area, collecting all the data from concentrator, computing management, control, print and inquiry of the data)[2], and its block diagram shown in Figure 1:

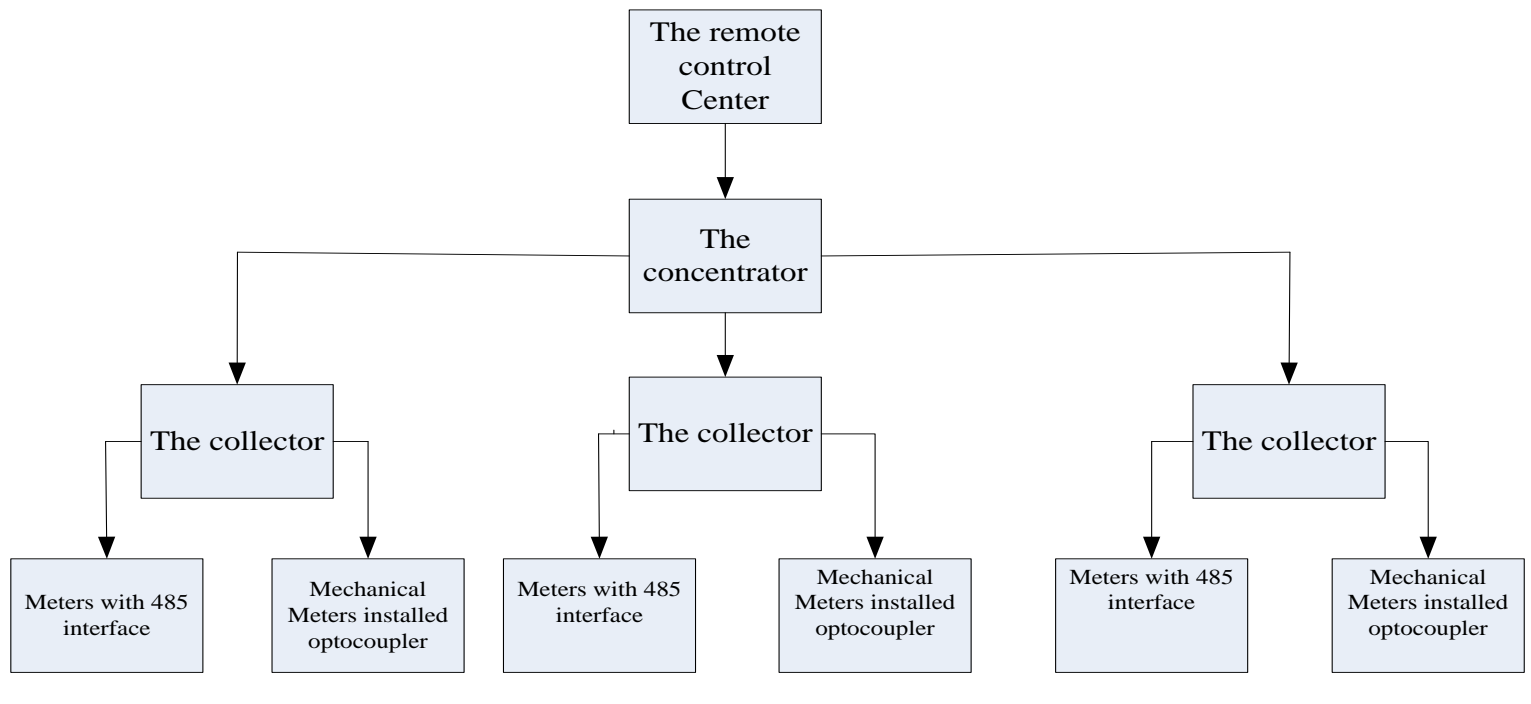

Fig.2 Overall design of the system structure

\section{The Collector}

Now many family's meters installed in the same meter box in the rural, so just using a collector in each meter box to collect all meters data. The collector uses AT89C52 as control chip, which can simultaneously collect up to sixteen meters data, and upload the data to the concentrator via the power line. Some of its peripheral circuit structure shown in Figure II: the backup battery of the power-down protection circuit can continue to supply the microcontroller for 48 hours when the system is powered down, which can effectively protect the 
integrity of the data. The watchdog circuit can reset the system effectively when the system works incorrectly, which improves the system's stability. The Microcontroller connects with meters with the 485 bus via the RS485 interface converter circuit, to complete the collection of the meter data. The power supply circuit generates the acquired $5 \mathrm{~V} \cdot 12 \mathrm{~V} \cdot 15 \mathrm{~V}$ voltage to ensure all the hardware circuit of the collector section to work properly. The status indication circuit works to indicate the working status of the collector [3]

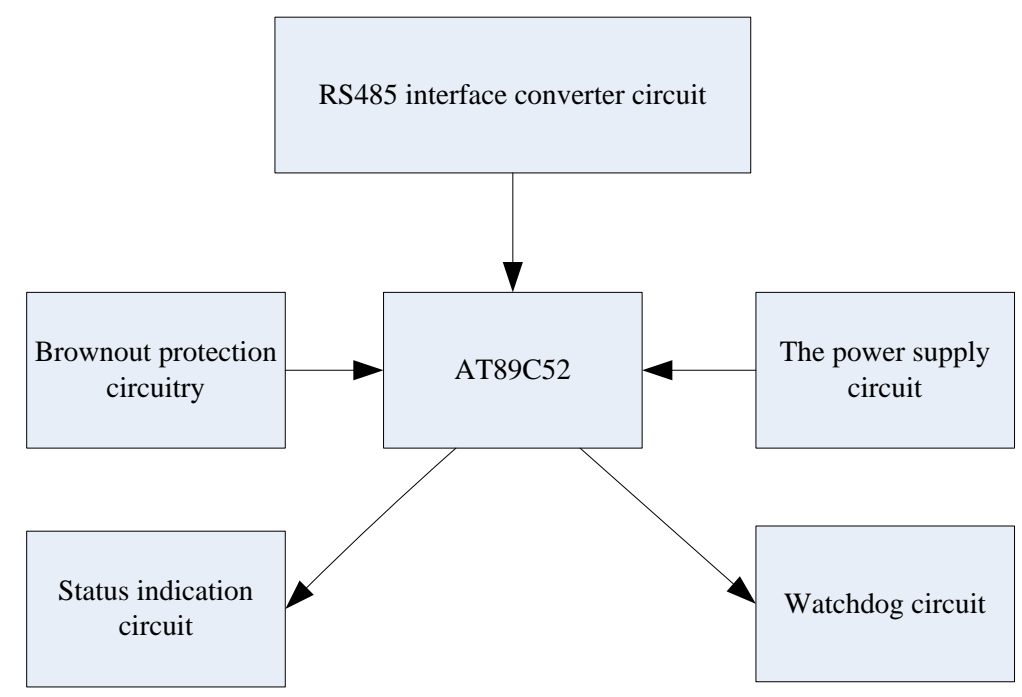

Fig.2 The Collector part of peripheral circuit structure diagram

The System uses low-voltage power line carrier communication, but because of the intermittent noise spikes in the field conditions, the random variation of the load impedance and strong attenuation of the signal, harsh conditions of the communication condition, so we must use a dedicated power line carrier communication chip to improve the above problems. SC1128 a chip that is integrated a spreading and dispreading, modulation and demodulation, D / $\mathrm{A}$ and $\mathrm{A} / \mathrm{D}$ conversion, the output drive, the input signal amplifier, watchdog, voltage detection module, and able to communicate with the microcontroller (MCU) via the serial port[4], reduces the cost and improves the function of the system.
SC1128 transmit or receive data through the port respectively named $\mathrm{SR}$, TX and $\mathrm{SYN}$. When $\mathrm{SR}$ equals zero, the circuit is in the receiving state; when $\mathrm{SR}=1$ the circuit is transmitting state. Its peripheral circuits are composed by the coupling circuit, band-pass filter circuit, PAMP amplifier circuit, amplifier circuit. Signal from the power line enters SC1128 via the coupler, large band-pass filter and amplifier circuit and after treatment of SC1128 goes into AT89C52. Data from AT89C52 is sent to the SC1128, after treatment of PAMP amplifier circuit and the coupling circuit, goes into the power line. This is the process of data sent and received on the power line. The working block diagram between Collector and SC1128 is shown in figure 3:

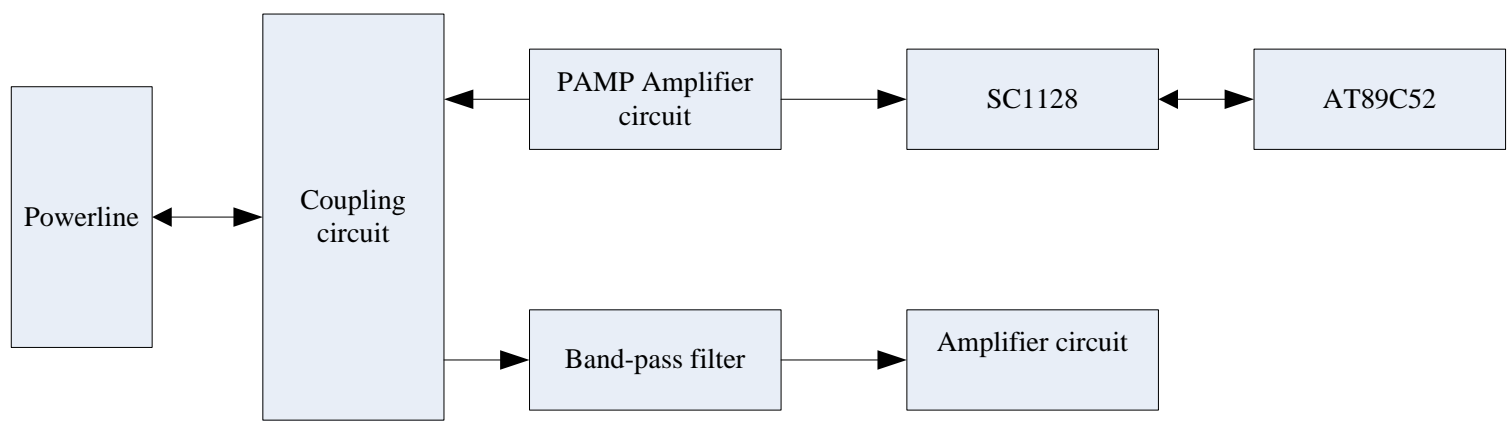

Fig.3 Logic diagram between collector and SC1128

\section{The Concentrator}

The Concentrator which uses AT89C52 as controller, connects to the remote control center using the existing GSM network via the GSM MODEM on upstream channel, [5] and connects Collector via power line carrier module on downstream channel. The Up / down channel uses a modular design, which is easy to use and to maintain and upgrade the system. The Concentrator communicate with the collector via 
power line carrier module and stores the meter data in the external memory; The Concentrator receives reading instruction from remote control centre via the GSM network in the form of short message and then transfer data from external memory to the remote control center and its structure diagram shown as Fig.4.

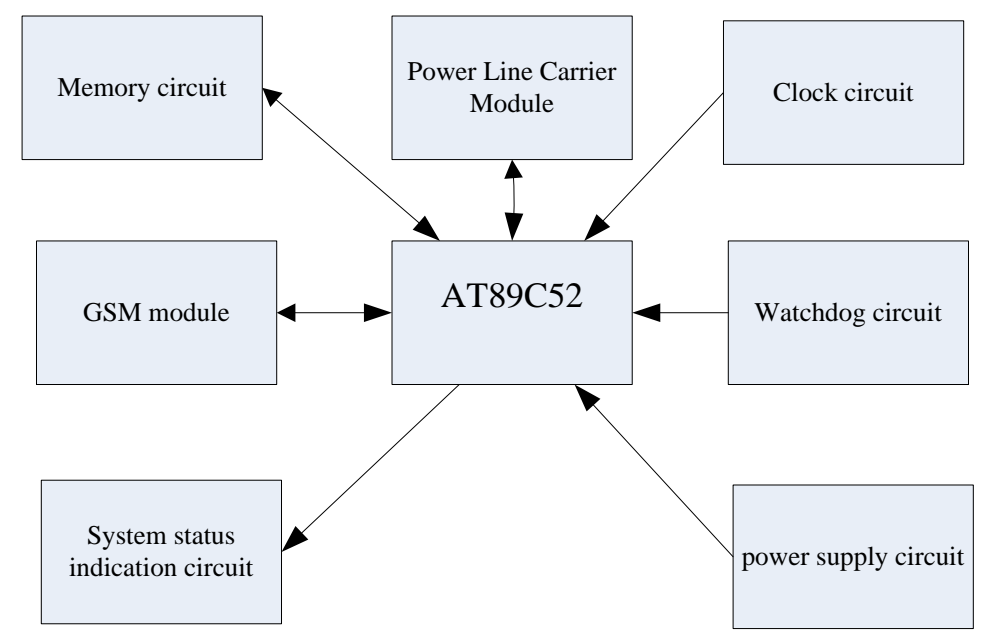

Fig.4 Logic diagram of Concentrator

\section{The Remote Control Center}

The Remote control center is responsible for the computing, management, control and print query tasks of data from all the concentrators in the electricity management region. The Management Center is located in the township substations, using several computers to communicate with each concentrator of various villages via GSM network, and completes receiving various data and control commands issued. The block diagram is shown in Figure 5.[6]

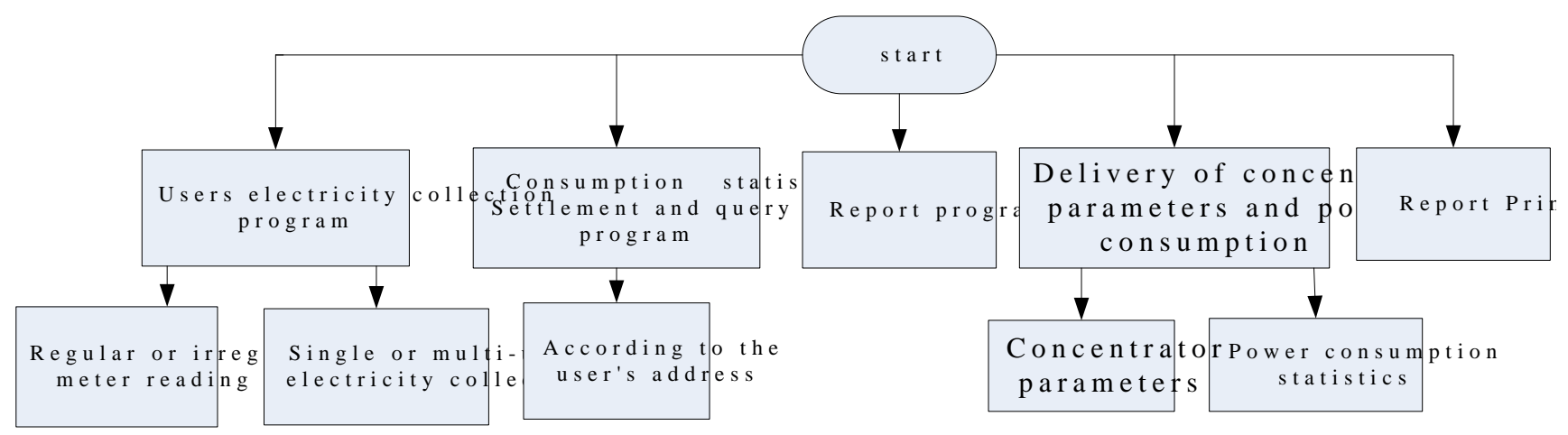

Fig.5 Logic diagram of remote control center

\section{The Conclusion}

The system using power line carrier chip SC1128 on upstream channel, makes communication using low-voltage power line between the collector and concentrator possible; the system downstream channel uses the nationwide GSM short message service, which makes the upper and lower channels no longer to lay cables, reducing the system costs. In addition, the system compared with the traditional manual meter reading system, improves the efficiency of real-time meter reading and the reliability of the data collecting, which adapts to the process of the development of power system automation and has a very broad application prospects.

\section{References}

[1] Yun Hu. Analysis and Study of Several Remote Meter Reading System. Low Voltage Apparatus, 2009 (8):28-29.

[2] Zhen Yang, Ge Zhang. The Forefront of the Industry. Study on Electric Power And Remote Automatic Meter Reading System, 2010, 10(4):3032.

[3] Hua Li, XiaoXin Sun. Practical Interface Technology of MCS-51 Series MCU Beijing University of Aeronautics and Astronautics Press, 1999

[4] Chong Qin. Research and Design of Power Line Carrier Communication System Based on SC1128 Chip, China New Technology and New Products, 2(2):143

[5] BinJie Han. Principle and Network Optimization of GSM. Mechanical Industry Press, 2002

[6] TingXia Liu, JiHong Xiu, JianHai Li. Intelligent Meter Reading System of Carrier Power Line, Electrical Automation. 2003, 3(6):61-64. 